\title{
Confinement, partial sleep deprivation and defined physical activity-influence on cardiorespiratory regulation and capacity
}

\author{
Jessica Koschate ${ }^{1}$ D . Uwe Drescher ${ }^{2} \cdot$ Uwe Hoffmann $^{3}$
}

Received: 10 January 2021 / Accepted: 13 May 2021 / Published online: 2 June 2021

(c) The Author(s) 2021

\begin{abstract}
Introduction Adequate cardiorespiratory fitness is of utmost importance during spaceflight and should be assessable via moderate work rate intensities, e.g., using kinetics parameters. The combination of restricted sleep, and defined physical exercise during a 45-day simulated space mission is expected to slow heart rate (HR) kinetics without changes in oxygen uptake $\left(\mathrm{V}_{2}\right)$ kinetics.

Methods Overall, 14 crew members ( 9 males, 5 females, $37 \pm 7 \mathrm{yrs}, 23.4 \pm 3.5 \mathrm{~kg} \mathrm{~m}^{-2}$ ) simulated a 45 -d-mission to an asteroid. During the mission, the sleep schedule included 5 nights of $5 \mathrm{~h}$ and 2 nights of $8 \mathrm{~h}$ sleep. The crew members were tested on a cycle ergometer, using pseudo-random binary sequences, changing between 30 and $80 \mathrm{~W}$ on day 8 before (MD-8), day 22 (MD22) and 42 (MD42) after the beginning and day $4(\mathrm{MD}+4)$ following the end of the mission. Kinetics information was assessed using the maxima of cross-correlation functions $\left(\mathrm{CCF}_{\max }\right)$. Higher $\mathrm{CCF}_{\max }$ indicates faster responses.

Results $\mathrm{CCF}_{\max }(\mathrm{HR})$ was significantly $(p=0.008)$ slower at MD-8 $(0.30 \pm 0.06)$ compared with MD22 $(0.36 \pm 0.06)$, MD42 $(0.38 \pm 0.06)$ and $\mathrm{MD}+4(0.35 \pm 0.06)$. Mean HR values during the different work rate steps were higher at MD- 8 and MD +4 compared to MD22 and MD42 $(p<0.001)$.

Discussion The physical training during the mission accelerated HR kinetics, but had no impact on mean HR values post mission. Thus, HR kinetics seem to be sensitive to changes in cardiorespiratory fitness and may be a valuable parameter to monitor fitness. Kinetics and capacities adapt independently in response to confinement in combination with defined physical activity and sleep.
\end{abstract}

Keywords Confinement $\cdot$ Heart rate regulation $\cdot$ Exercise training $\cdot$ Sleep restriction

Communicated by Ellen Adele Dawson.

Jessica Koschate

Jessica.Koschate@uni-oldenburg.de

Uwe Drescher

UweDrescher@gmx.de

Uwe Hoffmann

U.Hoffmann@dshs-koeln.de

1 Geriatric Medicine, Department for Health Services Research, School of Medicine and Health Sciences, Carl Von Ossietzky University Oldenburg, Ammerländer Heerstr. 140, 26129 Oldenburg, Germany

2 German Sport University Cologne, Am Sportpark Müngersdorf 6, 50933 Cologne, Germany

3 Institute of Exercise Training and Sport Informatics, Exercise Physiology, German Sport University Cologne, Am Sportpark Müngersdorf 6, 50933 Cologne, Germany

\begin{tabular}{|c|c|}
\hline \multicolumn{2}{|c|}{ Abbreviations } \\
\hline $\mathrm{ACF}$ & Auto-correlation function \\
\hline ANOVA & Analysis of variance \\
\hline CCFs & Cross-correlation function \\
\hline $\mathrm{CCF}_{\text {lag }}(\mathrm{x})$ & $\begin{array}{l}\text { Lag between the maximum of the ACF and } \\
\text { the CCF }\end{array}$ \\
\hline $\mathrm{CCF}_{\max }(\mathrm{x})$ & Maximum of the cross-correlation function \\
\hline HERA & Human exploration research analog \\
\hline HR & Heart rate \\
\hline HRV & Heart rate variability \\
\hline $\mathrm{mBP}$ & Mean blood pressure \\
\hline MD & Mission day \\
\hline NASA & $\begin{array}{l}\text { National aeronautics and space } \\
\text { administration }\end{array}$ \\
\hline PRBS & Pseudo random binary sequences \\
\hline$\dot{\mathrm{Q}}$ & Cardiac output \\
\hline SV & Stroke volume \\
\hline$\dot{\mathrm{V}}_{2}$ & Oxygen uptake \\
\hline$\dot{\mathrm{VO}}_{2 \text { musc }}$ & Muscular oxygen uptake \\
\hline
\end{tabular}




\begin{tabular}{|c|c|}
\hline$\dot{\mathrm{VO}}_{2 \text { peak }}$ & Peak oxygen uptake \\
\hline$\dot{\mathrm{VO}}_{2 \text { pulm }}$ & Pulmonary oxygen uptake \\
\hline VT & Ventilatory threshold \\
\hline $\mathrm{VT}_{1}$ & First ventilatory threshold \\
\hline WR & Work rate \\
\hline
\end{tabular}

\section{Introduction}

One of the main goals of life sciences in space research is to maintain the physical and cognitive fitness of individuals for future long-duration travels to the moon, Mars or even beyond (Strangman et al. 2014; Fomina et al. 2017; Norsk 2020). Safe, and time efficient diagnostic tools are needed to assess physical fitness in conditions without the possibility of medical interventions or close surveillance. Peak and/or maximal exercise performance [e.g., peak oxygen uptake capacity $\left(\dot{\mathrm{V}}_{2 \text { peak }}\right)$; maximal oxygen uptake $\left(\dot{\mathrm{VO}}_{2 \max }\right)$ ] (Hawkins et al. 2007; Poole and Jones 2017) as well as ventilatory thresholds (VTs) (Poole and Jones 2017) are usually assessed applying cardiopulmonary exercise tests and provide a comprehensive description of individual aerobic capacity and physical fitness. Another possibility to describe the functionality of the cardiorespiratory system is to analyze the response times (kinetics) of the different physiological parameters, e.g. heart rate (HR) and oxygen uptake $\left(\dot{\mathrm{VO}}_{2}\right)$ after changes in work rate (WR). The analysis of kinetics of the cardiorespiratory system offers valuable insights in cardiovascular and metabolic regulation (Hughson et al. 2001; Grassi 2000 2006; Rossiter 2011) and can be performed applying moderate WR intensities. One possibility to evaluate cardiorespiratory kinetics are WR protocols with sequences of pseudo randomly changing intensities (Hoffmann et al. 2013; Drescher et al. 2015; Koschate et al. 2016b). So-called pseudo random binary sequences (PRBS), as used by e.g., Hoffmann et al. (2013), are comparable to metabolic demands during every day habitual activities (Rossiter 2011). Since the assessment does not require high motivation or close medical surveillance, as would be the case for the assessment of $\mathrm{VO}_{2 \text { peak }}$ (Myers et al. 2014), the analysis of cardiorespiratory kinetics might be a valuable approach to monitor fitness during long duration missions to space, while supervision by medical staff is not directly available.

After prolonged missions to the International Space Station (ISS), a slowing of muscular oxygen uptake $\left(\dot{\mathrm{VO}}_{2 \mathrm{musc}}\right)$ kinetics was observed (Hoffmann et al. 2016). Additionally, the change in HR kinetics from pre- to post-flight was associated with changes in $\mathrm{V}_{2} \mathrm{O}_{2 \text { peak }}$. These decrements in physical performance during space flight are caused by the combination of microgravity, sleep problems (Barger et al. 2014; Yi et al. 2015; Mairesse et al. 2019), high and very controlled daily workloads as well as the confined living situation (De La Torre et al. 2012). Except for microgravity, these conditions can be simulated at the Lyndon B. Johnson Space Center of the National Aeronautics and Space Administration (NASA) in Houston, inside the Human exploration research analog (HERA) facility.

In former space analog conditions, using confined habitats, greater parasympathetic system activity during the wake time in the resting state was assessed throughout simulated space missions of 125 and $520 \mathrm{~d}$ (Vigo et al. 2012, 2013). After $70 \mathrm{~d}$ of confinement in a submarine, no changes in $\dot{\mathrm{VO}}_{2 \text { peak }}$, but an increase in the VT was reported in an exercise group, while decrements for the control group were observed in both parameters (Bennett et al. 1985).

Results from (partial) sleep deprivation experiments indicate a correlation between the onset of fatigue and heart rate variability (HRV) (Fogt et al. 2011), as well as higher sympathetic system activity, elevated autonomic stress and reduced HRV (Dettoni et al. 2012; Glos et al. 2014; Fogt et al. 2011; Liu et al. 2015), whereas after a fatiguing physical activity program, higher parasympathetic system activity was described (Jouanin et al. 2004). Greater global HRV in turn was associated with higher energy expenditure in everyday life and better perceived health status (Buchheit et al. 2005). HR kinetics seem to be very individual and react to the different physical and psychological stresses, the person experiences (Ludwig et al. 2018). To date, no information on HR kinetics, assessed during physical exercise as another indicator of neural control of HR, after sleep deprivation or during confinement is available. Since physical activity is restricted in a confined space, HR kinetics could be slowed, but if parasympathetic system activity is increased (Vigo et al. 2012, 2013), HR kinetics should be faster during isolation (Coote 2010).

The analysis of kinetics parameters during exercise can potentially help to monitor individual physical fitness during the isolation phase and may provide a tool for the estimation of performance in daily activities, which require an appropriate level of physical fitness.

On the basis of previous work in this field, the following hypotheses were investigated: the combination of restricted sleep and physical activity during 45 days of confinement (i) does not affect peak exercise capacity and $\dot{\mathrm{V}}_{2 \text { musc }}$ kinetics, but (ii) HR kinetics are slowed as a result of partial sleep deprivation in combination with the limited possibility to be physically active in the confined habitat.

\section{Methods}

\section{Participants}

The following inclusion criteria were applied: age range from 26 to 60 years, technical skills demonstrated through 
professional experience, motivation and work ethics similar to the current astronaut population, psychological qualification, at least bachelor's degree in engineering, biological science, physical science or mathematics. Ethical approval was obtained from the Institutional Review Board at the NASA Johnson Space Center (Protocol number: Pro2320) as well as the Ethical Committee of the German Sport University Cologne (Protocol number: 074/2016), and the experiments were conducted in accordance with the Declaration of Helsinki (including its amendments until 2013). Written informed consent was available from all participants prior to the experiments.

Overall, the HERA campaign four consisted of five $45 \mathrm{~d}$ missions, including four crew members each $(N=20)$. Due to Hurricane Harvey in August 2017, one mission with four participants had to be terminated early, and it was not possible to analyze these data sets. Further, data of two subjects could not be included for data analysis, because they had to terminate one of the exercise tests during the mission early. Both individuals exceeded the allowed HR or blood pressure values during the moderate exercise test protocol set by NASA medical board. Therefore, data of 14 individuals (9 males, 5 females) were included for statistical analyses. Anthropometric data of the subjects are shown in Table 1.

\section{Study outline}

The five 45-d-mission scenarios were identical and designed as follows: baseline data collection was performed over the two weeks prior to the isolation period. During the mission, the individuals simulated a mission to the asteroid 'Geographos' and back. Five consecutive nights per week they were restricted to five hours of sleep per night and during the weekends, two nights of eight hours of sleep were scheduled. During the missions three to five, an experimental lighting protocol was tested. One hour prior to sleep, the light was blue-depleted and two hours post sleep, the light was blue-enriched. Since only one mission $(n=4)$ was finished using the standard lighting protocol, the lighting procedures will not be included in the statistical analyses.
Every second day, the crew exercised either on a cycle ergometer or they performed toning and stretching exercises over a time period of $30 \mathrm{~min}$, while the allowed HR during exercise was restricted to below $85 \%$ of their age adjusted maximum.

The subjects filled in a questionnaire about the general frequency of their exercising habits and their physical activities during the year before the first exercise test prior to the beginning of the mission.

\section{Exercise test protocol}

Before, during and after the mission, a standardized exercise test was applied to determine the kinetics responses in the moderate exercise range. The tests were scheduled eight days before the start of the mission (MD-8), on day 22 (MD22) and day 42 (MD42) of the mission, as well as four days after the mission ended $(\mathrm{MD}+4)$. Eight hours ahead of the exercise test, the subjects stopped to consume alcohol and nicotine eight hours before, they stopped drinking caffeine four hours prior to the test and they started fasting two hours ahead of the beginning of the test. Additionally, eight hours before the test, no exercise was permitted and $24 \mathrm{~h}$ prior to the test session no maximal exercise was allowed. The exercise tests on MD22 were scheduled on day five of a sleep restriction period and MD42 was day four of a sleep restriction phase.

The exercise test was performed on a cycle ergometer (Lode Corival, Lode BV, Groningen, The Netherlands) and consisted of two parts: the first part started with the resting condition, followed by a $300 \mathrm{~s}$ phase at a low constant exercise intensity $(30 \mathrm{~W})$, which was followed by two PRBS with dynamic WR changes (30 and $80 \mathrm{~W}$ ) for $300 \mathrm{~s}$ each. Thereafter, a constant phase of the higher WR $(80 \mathrm{~W})$ was applied for $300 \mathrm{~s}$. The second part of the WR protocol was only performed on MD- 8 and MD +4 . On these days, the WR was further increased to $100 \mathrm{~W}$ for one minute and afterwards WR was increased by $25 \mathrm{~W}$ every minute until fatigue (Fig. 1A).
Table 1 Anthropometric data of the crew

\begin{tabular}{|c|c|c|c|c|c|c|c|c|}
\hline \multirow[t]{2}{*}{$N=14$} & \multicolumn{2}{|l|}{ MD-8 } & \multicolumn{2}{|l|}{ MD22 } & \multicolumn{2}{|l|}{ MD42 } & \multicolumn{2}{|c|}{$\mathrm{MD}+4$} \\
\hline & Mean & SD & Mean & SD & Mean & SD & Mean & SD \\
\hline Age (years) & 37 & 7 & & & & & & \\
\hline Height $(\mathrm{cm})$ & 176 & 9 & & & & & & \\
\hline Body mass (kg) & 73.4 & 14.6 & 72.3 & 13.8 & 72.3 & 13.8 & 72.3 & 13.5 \\
\hline BMI $\left(\mathrm{kg} \mathrm{m}^{2}\right)$ & 23.4 & 3.5 & 23.3 & 2.9 & 23.1 & 3.0 & 23.1 & 3.0 \\
\hline
\end{tabular}

$M D-88$ days before the beginning of the mission, $M D 22$ mission day 22, $M D 42$ mission day $42, M D+4$ 4 days after the end of the mission 
During all tests, gas exchange was measured breathby-breath, using a metabolic cart (Metalyzer 3B, Cortex Biophysik GmbH, Leipzig, Germany), including the corrections of Beaver et al. (1981) for alveolar gas exchange. The metabolic cart was calibrated according to the manufacturer's guidelines, prior to all tests. HR was measured using an ECG (CustoGuard belt 3, CustoMed, Ottobrunn, Germany) and blood pressure was recorded beat-tobeat via Portpares Model 2 (Finapres Medical Systems, Amsterdam, The Netherlands). Using the blood pressure values, stroke volume (SV) was calculated applying the Modelflow Algorithm of the beatscope software (Finapres Medical Systems, Amsterdam, The Netherlands), considering age, height and body mass.
Breath-by-breath data were interpolated step-wise and beat-to-beat data linearly to 1 -s-intervals to obtain homogenous sampling.

Peak data of $\mathrm{VO}_{2}$, HR and WR before and after the mission are reported as averages over the last $30 \mathrm{~s}$ before the termination of the WR protocol. The first VT $\left(\mathrm{VT}_{1}\right)$ was determined using the $\mathrm{V}$-slope method in combination with the ventilatory equivalents and endtidal values of $\mathrm{O}_{2}$ and $\mathrm{CO}_{2}$, as suggested by Beaver et al. (1986).

To receive the kinetics information of the data, timeseries analysis was applied. The PRBS signal was autocorrelated (ACF) and interpreted as a WR impulse (Bennett et al. 1981). The Cross-correlation functions (CCFs) between WR and the respective parameters as i.e., $\mathrm{HR}$ and pulmonary $\dot{\mathrm{VO}}_{2}\left(\dot{\mathrm{VO}}_{2 \text { pulm }}\right)$ were calculated and interpreted as
Fig. 1 Description of the work rate (WR) protocol (A) and the kinetics analysis (B) before, during and after the mission. $H R$ heart rate, $\dot{\mathrm{V}} \mathrm{O}_{2 \text { pulm }}$ pulmonary oxygen uptake; $\dot{V} O_{2 m u s c}$ muscular oxygen uptake, $M D-8$ day 8 before the start of the mission, $M D+4$ four days after the end of the mission, $C C F_{\max }(x)$ maximum of the cross-correlation function between WR and the respective parameter (x), $C C F(x)$ cross-correlation function between WR and the respective parameter $(\mathrm{x})$

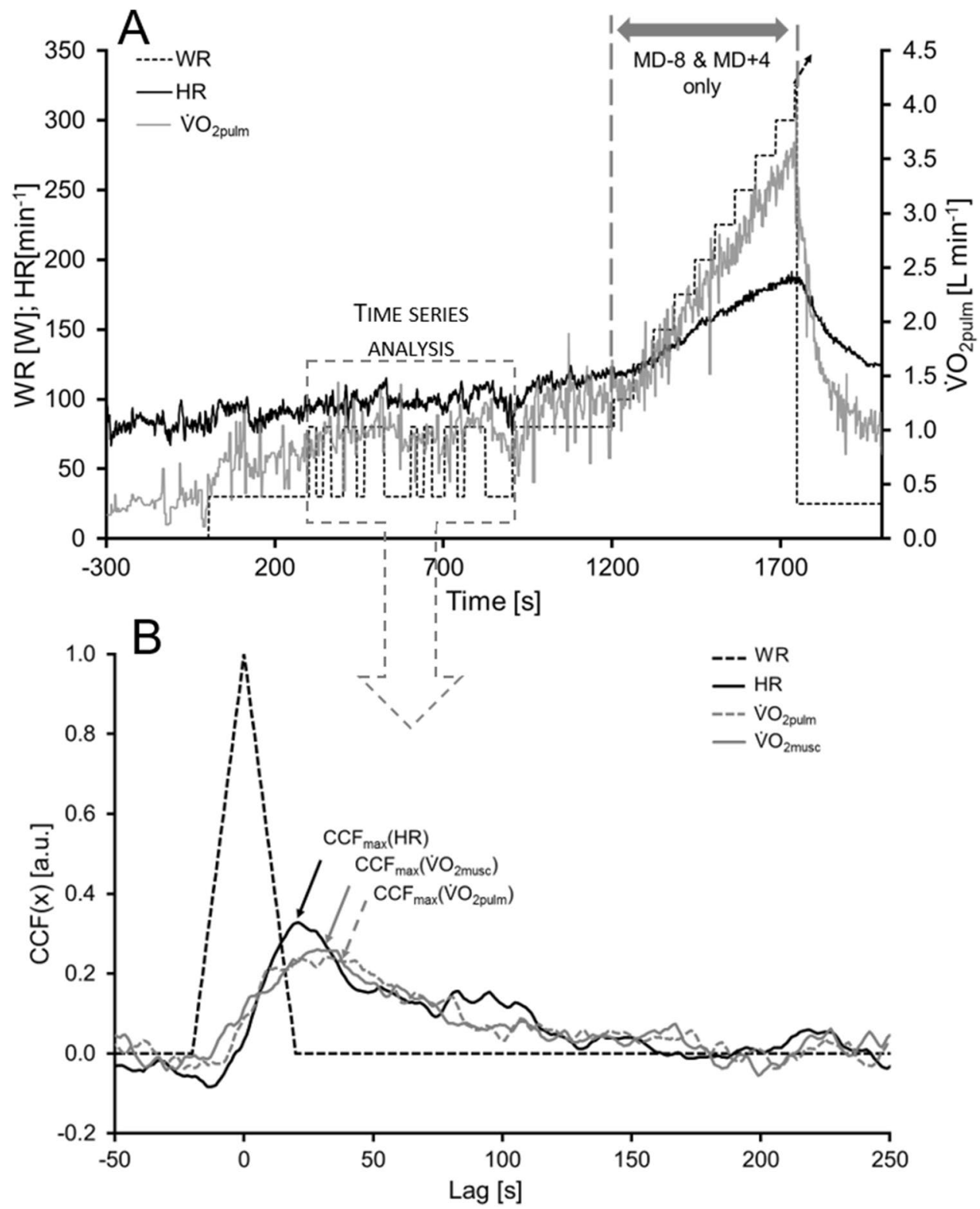


the parameters' response to this WR impulse. The higher the maximum of the $\mathrm{CCF}\left(\mathrm{CCF}_{\max }\right)$, the faster was the kinetics response. The greater the lag between the $\mathrm{CCF}_{\max }$ and the maximum of the $\mathrm{ACF}\left(\mathrm{CCF}_{\mathrm{lag}}\right)$, the more time delayed was the onset of the response (compare Fig. 1B). Muscular $\mathrm{V}_{2}$ $\left(\mathrm{V}_{2 \text { musc }}\right.$ ) was estimated from $\mathrm{HR}$ and $\dot{V} \mathrm{O}_{2 \text { pulm }}$, using the approach of Hoffmann et al. (2013). In this method, a specific venous volume in combination with $\dot{\mathrm{VO}}_{2}$ and perfusion of the remainder of the body is estimated to calculate transit times of the $\mathrm{V}_{2 \text { musc }}$ signal from muscle to mouth.

\section{Statistical analysis}

Normal distribution of the respective parameters was tested applying the Kolmogorov-Smirnov test. If normal distribution could be assumed, analysis of variance (ANOVA) with repeated measures on the factor day (MD-8, MD22, MD42, MD + 4) and Bonferroni post hoc tests were calculated for the $\mathrm{CCF}_{\text {lag }}$ and $\mathrm{CCF}_{\text {max }}$ values. In case normal distribution could not be assumed, Friedman tests with Wilcoxon post hoc tests were used and the False Discovery Rate correction procedure (Benjamini and Hochberg 1995) for multiple testing was applied. ANOVA with the factors day (MD-8, MD22, MD42, MD +4) and step [Rest, $30 \mathrm{~W}$, $53.3 \mathrm{~W}$ (2xPRBS), $80 \mathrm{~W}$ ] with repeated measures on both factors were applied to compare the mean values of $\mathrm{HR}$, $\dot{\mathrm{V}}_{2 \text { pulm }}$, mean arterial blood pressure (mBP), SV and cardiac output ( $\dot{\mathrm{Q}})$. In case sphericity could not be assumed, the Huynh-Feldt correction was used to assess the inner subject effects. Bonferroni post hoc tests were applied, if appropriate. The Pearson product-moment correlation coefficient (one-tailed) was calculated for normally distributed data to test for correlations. The level of significance was set to $\alpha=5 \%$. All statistical tests were performed using SPSS 26 (IBM, Amonk, New York, USA).

\section{Results}

The results of the peak exercise test before and after the mission are shown in Table 2.

Over the 12 months prior to the start of the mission, the participants exercised $15 \pm 6$ times per month and about $46 \pm 33$ min each day.

Comparing the mean values during the different WR steps of the exercise protocol, $\mathrm{HR}, \mathrm{V}_{2 \text { pulm }}, \mathrm{mBP}$ and $\mathrm{SV}$, but not Q showed significant differences over time (see Table 3). Only HR showed a significant interaction for time $\times$ step (post hoc results are shown in Table 3). Independent of the steps during the WR protocol, $\dot{\mathrm{V}}_{2 \text { pulm }}$ was significantly higher at MD-8 compared with MD22 $(p=0.009)$ and MD42 $(p=0.003)$. Similarly, mBP values were significantly higher at MD-8 in comparison with MD22 $(p=0.028)$ and MD42
Table 2 Peak values before and after the mission as well as exercise habits before the mission

\begin{tabular}{|c|c|c|c|c|c|}
\hline \multirow[t]{2}{*}{$N=14$} & \multicolumn{2}{|l|}{ MD-8 } & \multicolumn{2}{|c|}{$\mathrm{MD}+4$} & \multirow[t]{2}{*}{ Sig } \\
\hline & Mean & SD & Mean & SD & \\
\hline $\mathrm{WR}_{\text {peak }}(\mathrm{W})$ & 257 & 63 & 259 & 56 & 0.661 \\
\hline $\mathrm{HR}_{\text {peak }}\left(\min ^{-1}\right)$ & 180 & 12 & 179 & 12 & 0.506 \\
\hline$\dot{V} \mathrm{CO}_{2 \text { peak }}\left(\mathrm{L} \mathrm{min}^{-1}\right)$ & 3.45 & 0.97 & 2.82 & 0.62 & 0.747 \\
\hline$\dot{V} \mathrm{O}_{2 \text { peak }}\left(\mathrm{ml} \mathrm{kg}^{-1} \min ^{-1}\right)$ & 37.79 & 5.85 & 38.92 & 4.62 & 0.221 \\
\hline $\operatorname{RER}_{\text {peak }}$ (a.u.) & 1.23 & 0.07 & 1.21 & 0.06 & 0.486 \\
\hline WR @ VT $1(\mathrm{~W})$ & 163 & 34 & 182 & 36 & 0.033 \\
\hline $\mathrm{HR} @ \mathrm{VT}_{1}\left(\mathrm{~min}^{-1}\right)$ & 156 & 19 & 156 & 13 & 0.826 \\
\hline$\dot{V} \mathrm{O}_{2} @ \mathrm{VT}_{1}\left(\mathrm{~L} \mathrm{~min}^{-1}\right)$ & 1.92 & 0.35 & 2.04 & 0.44 & 0.135 \\
\hline
\end{tabular}

$M D-88$ days before the beginning of the mission, $M D+44$ days after the end of the mission, $V T_{1}$ first ventilatory threshold, $W R_{\text {peak }}$ peak work rate, $H R_{\text {peak }}$ peak heart rate, $\dot{V} C O_{2 p e a k}$ peak carbon dioxide output, $\dot{V} O_{2 \text { peak }}$ peak oxygen uptake, $R E R_{\text {peak }}$ peak respiratory exchange ratio, WR @VT 1 work rate at the first ventilatory threshold, $H R$ @ $V T_{1}$ heart rate at the first ventilatory threshold, $\dot{V} O_{2} @ V T_{1}$ oxygen uptake at the first ventilatory threshold

$(p=0.007)$. At MD-8, SV was significantly lower compared with MD42 $(p=0.031)$. Further post hoc results of the interaction effects (time $\times$ step) are shown in Table 3 .

Comparing the $\mathrm{CCF}_{\text {max }}$ and $\mathrm{CCF}_{\text {lag }}$ values of $\mathrm{VO}_{2 \text { musc }}$, $\mathrm{HR}, \dot{\mathrm{V}} \mathrm{O}_{2 \text { pulm }}$ and $\mathrm{mBP}$ between the different mission days, significant differences were observed for $\mathrm{CCF}_{\max }(\mathrm{HR})$ only $(p=0.008)$. Post hoc results are shown in Table 4.

Significant correlations were found between the change in $\dot{\mathrm{VO}}_{2 \text { peak }}$ from $\mathrm{MD}-8$ to $\mathrm{MD}+4$ and the baseline $\dot{\mathrm{V}} \mathrm{O}_{2 \text { peak }}$ values at MD-8 ( $r=-0.614, p=0.010$; Fig. 2$)$.

Further, the change in $\mathrm{CCF}_{\max }\left(\mathrm{V}_{2 \text { musc }}\right)$ from MD-8 to $\mathrm{MD}+4$ correlated significantly with the baseline value (MD-8) of $\mathrm{CCF}_{\text {max }}\left(\mathrm{V} \mathrm{O}_{2 \text { musc }}\right)(r=-0.763, p=0.001$; Fig. 3).

\section{Discussion}

The aim of the experiment was to show the effect of 45 days of confinement in combination with the prescribed exercise training and sleep restrictions on parameters of exercise capacity and cardiorespiratory regulation. In accordance with the proposed hypothesis, (i) neither $\dot{\mathrm{VO}}_{2 \text { peak }}$ nor $\dot{\mathrm{V}} \mathrm{O}_{2 \text { musc }}$ kinetics changed significantly over the different test days during the HERA C4 missions. Therefore, the combination of confinement and sleep restrictions did not affect overall exercise capacity and the regulation of $\dot{\mathrm{VO}}_{2 \text { musc }}$. However, $\mathrm{CCF}_{\max }(\mathrm{HR})$ was higher during and after the mission, when compared with the values before the mission. Hence, HR kinetics can be interpreted as faster during and after the simulated space mission, which is in contrast to the established hypothesis of slower HR kinetics as a result of the combination of partial sleep deprivation and restricted 
Table 3 Means and standard deviations for the different steps of the work rate protocol

\begin{tabular}{|c|c|c|c|c|c|c|c|c|c|c|c|c|c|c|c|c|}
\hline \multirow[t]{2}{*}{$N=14$} & \multirow[t]{2}{*}{ Phase } & \multicolumn{2}{|l|}{ MD-8 } & & \multicolumn{2}{|c|}{ MD22 } & & \multicolumn{2}{|c|}{ MD42 } & & \multicolumn{2}{|c|}{$\mathrm{MD}+4$} & & \multirow[t]{2}{*}{ Time } & \multirow[t]{2}{*}{ Step } & \multirow[t]{2}{*}{ Time $\times$ step } \\
\hline & & Mean & SD & & Mean & SD & & Mean & SD & & Mean & SD & & & & \\
\hline \multirow[t]{5}{*}{$\mathrm{HR}\left(\min ^{-1}\right)$} & Rest & 86 & 11 & \multirow[b]{2}{*}{$\mathrm{a}, \mathrm{b}$} & 80 & 8 & & 79 & 14 & & 86 & 10 & & \multirow[t]{5}{*}{$<0.001$} & \multirow[t]{5}{*}{$<0.001$} & \multirow[t]{5}{*}{0.009} \\
\hline & 30 & 97 & 13 & & 88 & 11 & $c, d$ & 87 & 11 & $c, d$ & 95 & 11 & $\mathrm{a}, \mathrm{b}$ & & & \\
\hline & 53.3 & 109 & 15 & $\mathrm{a}, \mathrm{b}$ & 97 & 13 & $c, d$ & 96 & 11 & $\mathrm{c}, \mathrm{d}$ & 105 & 13 & $\mathrm{a}, \mathrm{b}$ & & & \\
\hline & 53.3 & 113 & 17 & $\mathrm{a}, \mathrm{b}$ & 99 & 14 & $c, d$ & 99 & 13 & $\mathrm{c}, \mathrm{d}$ & 109 & 14 & $\mathrm{a}, \mathrm{b}$ & & & \\
\hline & 80 & 130 & 21 & a, b & 111 & 13 & $c, d$ & 111 & 13 & $\mathrm{c}, \mathrm{d}$ & 125 & 18 & $\mathrm{a}, \mathrm{b}$ & & & \\
\hline \multirow[t]{5}{*}{$\dot{V} \mathrm{O}_{2 \text { pulm }}\left(\mathrm{L} \mathrm{min}^{-1}\right)$} & Rest & 0.37 & 0.08 & & 0.36 & 0.09 & & 0.33 & 0.06 & & 0.37 & 0.08 & & \multirow[t]{5}{*}{$<0.001$} & \multirow[t]{5}{*}{$<0.001$} & \multirow[t]{5}{*}{0.758} \\
\hline & 30 & 0.75 & 0.08 & $\mathrm{~b}$ & 0.71 & 0.07 & & 0.69 & 0.09 & c & 0.72 & 0.07 & & & & \\
\hline & 53.3 & 0.95 & 0.08 & $\mathrm{a}, \mathrm{b}$ & 0.90 & 0.06 & c & 0.89 & 0.06 & $\mathrm{c}, \mathrm{d}$ & 0.93 & 0.07 & $\mathrm{~b}$ & & & \\
\hline & 53.3 & 0.97 & 0.07 & a, b & 0.92 & 0.06 & c & 0.90 & 0.06 & c & 0.94 & 0.08 & & & & \\
\hline & 80 & 1.25 & 0.07 & b & 1.14 & 0.20 & & 1.13 & 0.17 & $\mathrm{c}$ & 1.21 & 0.11 & & & & \\
\hline \multirow[t]{5}{*}{ mBP (mmHg) } & Rest & 95 & 14 & & 89 & 12 & & 87 & 8 & & 91 & 9 & & \multirow[t]{5}{*}{0.001} & \multirow[t]{5}{*}{$<0.001$} & \multirow[t]{5}{*}{0.087} \\
\hline & 30 & 105 & 12 & $\mathrm{a}, \mathrm{b}$ & 90 & 12 & c & 89 & 6 & c & 94 & 10 & & & & \\
\hline & 53.3 & 105 & 12 & $\mathrm{a}, \mathrm{b}$ & 93 & 12 & c & 92 & 7 & c & 96 & 10 & & & & \\
\hline & 53.3 & 104 & 11 & $\mathrm{a}, \mathrm{b}$ & 92 & 10 & c & 90 & 8 & $\mathrm{c}$ & 95 & 9 & & & & \\
\hline & 80 & 105 & 13 & $\mathrm{a}, \mathrm{b}$ & 93 & 11 & c & 93 & 9 & $c, d$ & 99 & 9 & $\mathrm{~b}$ & & & \\
\hline \multirow[t]{5}{*}{$\mathrm{SV}(\mathrm{ml})$} & Rest & 68 & 19 & & 68 & 14 & & 73 & 19 & & 75 & 15 & & \multirow[t]{5}{*}{0.045} & \multirow[t]{5}{*}{$<0.001$} & \multirow[t]{5}{*}{0.227} \\
\hline & 30 & 82 & 31 & & 96 & 26 & & 97 & 34 & & 92 & 18 & & & & \\
\hline & 53.3 & 87 & 32 & $\mathrm{~b}$ & 99 & 24 & & 108 & 35 & c & 97 & 21 & & & & \\
\hline & 53.3 & 91 & 30 & & 102 & 25 & & 105 & 28 & & 96 & 19 & & & & \\
\hline & 80 & 94 & 31 & & 99 & 27 & & 102 & 35 & & 101 & 19 & & & & \\
\hline \multirow[t]{5}{*}{$\dot{Q}\left(\mathrm{~L} \mathrm{~min}^{-1}\right)$} & Rest & 5.83 & 1.53 & & 5.39 & 1.29 & & 5.79 & 2.12 & & 6.27 & 1.41 & & 0.652 & $<0.001$ & 0.296 \\
\hline & 30 & 7.89 & 2.85 & & 8.44 & 2.42 & & 8.35 & 3.00 & & 8.26 & 2.88 & & & & \\
\hline & 53.3 & 9.31 & 2.88 & & 9.55 & 2.62 & & 10.25 & 3.16 & & 9.91 & 2.80 & & & & \\
\hline & 53.3 & 10.02 & 2.73 & & 9.96 & 2.46 & & 10.23 & 2.54 & & 10.37 & 2.36 & & & & \\
\hline & 80 & 11.97 & 3.35 & & 10.91 & 3.15 & & 11.10 & 3.37 & & 12.36 & 2.54 & & & & \\
\hline
\end{tabular}

Phase phase of the test protocol, $W R$ work rate, $H R$ heart rate, $\dot{V} \mathrm{O}_{2 \text { pulm }}$ pulmonary oxygen uptake, $m B P$ mean arterial pressure, $S V$ stroke volume, $\dot{Q}$ cardiac output, $M D-88$ days before the beginning of the isolation period, $M D 22$ Day 22 of the isolation period, $M D 42$ Day 42 of the isolation period, $M D+44$ days after end of the isolation period

${ }^{\text {a }}$ Significantly different to MD22

${ }^{\mathrm{b}}$ Significantly different to MD42

${ }^{\mathrm{c}}$ Significantly different to MD-8

${ }^{\mathrm{d}}$ Significantly different to MD +4

physical activity during the confinement phase (ii). Further, absolute values of HR were higher before and after the mission, compared with the values during the mission.

\section{Oxygen uptake-peak values and its regulation}

The $\dot{\mathrm{V}}_{2 \text { peak }}$ values did not change significantly from MD- 8 to MD +4 . Therefore, the applied exercise program was sufficient to mitigate the potentially deconditioning effects of lower physical activity throughout the $45 \mathrm{~d}$ of confinement in combination with sleep restrictions. However, the small but significant increase in WR of $20 \mathrm{~W}$ at $\mathrm{VT}_{1}$ after the end of the mission $(\mathrm{MD}+4)$, suggests a slightly increased exercise tolerance. Similar results were found for a submarine crew, living in confinement for $70 \mathrm{~d}$. $\dot{\mathrm{V}} \mathrm{O}_{2 \text { peak }}$ did not change significantly, but $\mathrm{VT}_{1}$ as a percentage of $\dot{\mathrm{VO}}_{2 \text { peak }}$ increased after 8 weeks of endurance exercise training, which is comparable to the effects of the exercise training program applied during HERA C4 (Bennett et al. 1985). However, correlation analyses revealed, that HERA crew members with greater $\dot{\mathrm{VO}}_{2 \text { peak }}$ and faster $\dot{\mathrm{V}}_{2 \text { musc }}$ kinetics before the mission showed greater declines in the respective parameters after the mission. Moraes et al. (2018), analyzing $\dot{\mathrm{VO}}_{2 \text { peak }}$ values of differently trained crew members (mountaineers and scientists) before and after an Antarctic field expedition reported comparable findings. Similar results were reported for Astronauts during spaceflight (Moore et al. 2014): the $\dot{\mathrm{V}} \mathrm{O}_{2 \text { peak }}$ did not decrease in all crew members, 
Table 4 Kinetics responses during the exercise test

\begin{tabular}{|c|c|c|c|c|c|c|c|c|c|c|}
\hline \multirow[t]{2}{*}{$N=14$} & & \multicolumn{2}{|l|}{ MD-8 } & \multicolumn{2}{|c|}{ MD22 } & \multicolumn{2}{|c|}{ MD42 } & \multicolumn{2}{|c|}{$\mathrm{MD}+4$} & \multirow{2}{*}{$\begin{array}{l}\text { Sig. for } \\
\text { factor } \\
\text { time }\end{array}$} \\
\hline & & Mean & SD & Mean & SD & Mean & SD & Mean & SD & \\
\hline \multirow[t]{2}{*}{$\dot{V} \mathrm{O}_{2 \text { musc }}$} & $\mathrm{CCF}_{\max }$ [a.u.] & 0.35 & 0.05 & 0.35 & 0.05 & 0.38 & 0.06 & 0.35 & 0.05 & 0.354 \\
\hline & $\mathrm{CCF}_{\text {lag }}[\mathrm{s}]$ & 22 & 10 & 26 & 9 & 22 & 10 & 25 & 14 & 0.770 \\
\hline \multirow[t]{2}{*}{ HR } & $\mathrm{CCF}_{\max }$ [a.u.] & $0.30^{\mathrm{b}, \mathrm{c}, \mathrm{d}}$ & 0.06 & $0.36^{\mathrm{a}}$ & 0.06 & $0.38^{\mathrm{a}}$ & 0.06 & $0.35^{\mathrm{a}}$ & 0.06 & 0.008 \\
\hline & $\mathrm{CCF}_{\text {lag }}[\mathrm{s}]$ & 24 & 29 & 19 & 6 & 26 & 21 & 21 & 18 & 0.142 \\
\hline \multirow[t]{2}{*}{$\dot{V} \mathrm{O}_{2 \text { pulm }}$} & $\mathrm{CCF}_{\max }$ [a.u.] & 0.35 & 0.06 & 0.35 & 0.06 & 0.35 & 0.04 & 0.34 & 0.05 & 0.781 \\
\hline & $\mathrm{CCF}_{\text {lag }}[\mathrm{s}]$ & 31 & 14 & 33 & 7 & 30 & 11 & 36 & 15 & 0.326 \\
\hline \multirow[t]{2}{*}{$\mathrm{mBP}$} & $\mathrm{CCF}_{\max }$ [a.u.] & 0.25 & 0.07 & 0.28 & 0.09 & 0.26 & 0.05 & 0.23 & 0.04 & 0.216 \\
\hline & $\mathrm{CCF}_{\text {lag }}[\mathrm{s}]$ & 108 & 73 & 116 & 94 & 73 & 77 & 88 & 86 & 0.653 \\
\hline
\end{tabular}

$\dot{V} \mathrm{O}_{2 \text { musc }}$ muscular oxygen uptake, $H R$ heart rate, $\dot{V} \mathrm{O}_{2 \text { pulm }}$ pulmonary oxygen uptake, $m B P$ mean arterial pressure, $M D-88$ days before the beginning of the isolation period, $M D 22$ Day 22 of the isolation period, $M D 42$ Day 42 of the isolation period, $M D+44$ days after the end of the mission, Sig significant effect

${ }^{\text {a }}$ Significantly different to MD-8 $(p \leq 0.05)$

${ }^{\mathrm{b}}$ Significantly different to MD22 $(p \leq 0.05)$

${ }^{\mathrm{c}}$ Significantly different to MD42 $(p \leq 0.05)$

${ }^{\mathrm{d}}$ Significantly different to MD+4 $(p \leq 0.05)$

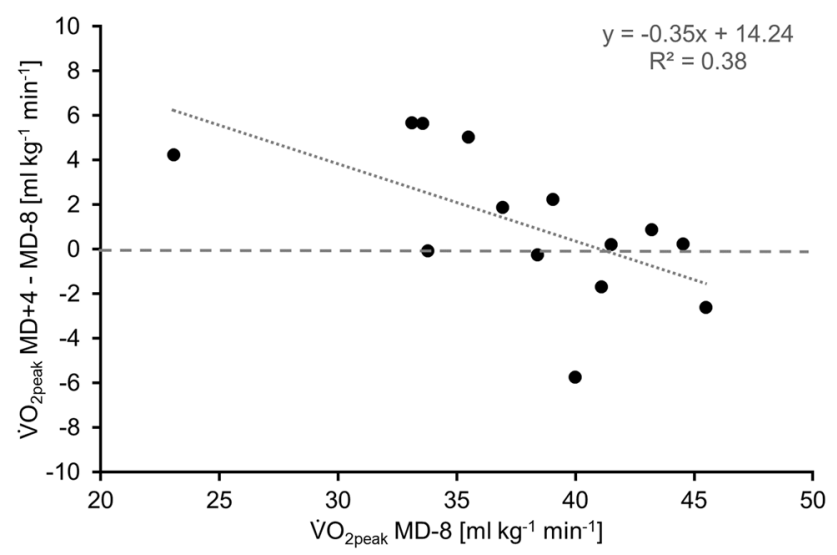

Fig. 2 Correlation $(r=-0.614, p=0.010)$ between peak oxygen uptake $\left(\dot{\mathrm{VO}}_{2 \text { peak }}\right)$ on MD-8 and the difference in $\dot{\mathrm{V}} \mathrm{O}_{2 \text { peak }}$ from pre to post test. Negative values on the ordinate indicate decreases in $\dot{\mathrm{V}} \mathrm{O}_{2 \text { peak }}$ from pre to post test $(N=14)$. MD-8: 8 days before the beginning of the mission, $\mathrm{MD}+4: 4$ days after the end of the mission

but those starting with higher levels of $\dot{\mathrm{VO}}_{2 \text { peak }}$ were more prone to losses at the first inflight test, and those who maintained their preflight $\dot{\mathrm{V}} \mathrm{O}_{2 \text { peak }}$ values after return to Earth, spent about $80 \%$ of their inflight aerobic training time at $~ 80 \%$ of their maximum HR (Moore et al. 2014). For the HERA crew, training prescriptions should have been more customized for the individual training status of the crew members, to apply individually adequate training stimuli throughout the mission phase. Highly trained

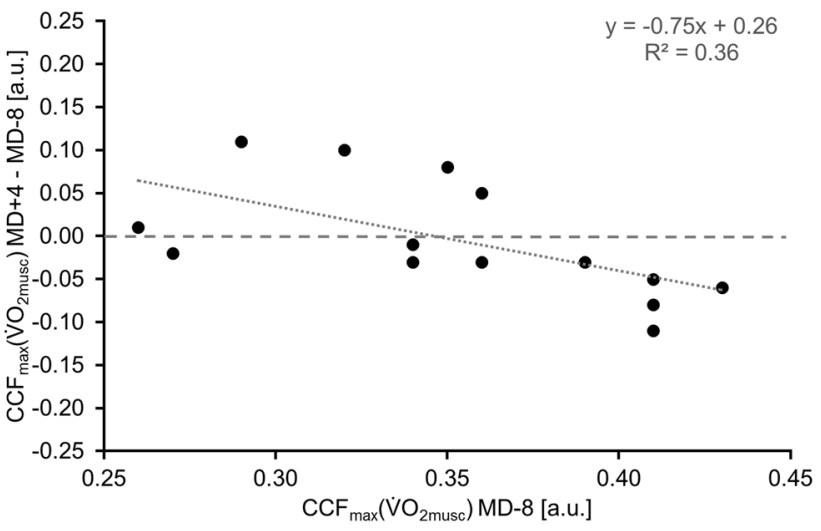

Fig. 3 Correlation $(r=-0.641, p=0.007)$ between muscular oxygen uptake kinetics $\left(\mathrm{CCF}_{\max }\left(\dot{\mathrm{VO}}_{2 \text { musc }}\right)\right)$ on MD-8 and the difference in $\mathrm{CCF}_{\text {max }}\left(\dot{\mathrm{V}}_{2 \text { musc }}\right)$ from pre to post test. Negative values on the ordinate indicate lower $\mathrm{CCF}_{\max }\left(\dot{\mathrm{VO}}_{2 \text { musc }}\right)$ from pre to post test $(N=14)$. MD-8: 8 days before the beginning of the mission, $M D+4: 4$ days after the end of the mission

individuals need a higher training volume to sustain their personal fitness level, which is also known from longitudinal ageing studies (Katzel et al. 2001).

The training effect, indicated by an increased $\mathrm{WR}$ at $\mathrm{VT}_{1}$ did not result in faster $\mathrm{V}_{2 \text { musc }}$ kinetics. This suggests different timelines for the adjustment of peak exercise capacities $\left(\dot{\mathrm{VO}}_{\text {2peak }}\right)$, submaximal aerobic capacities $\left(\mathrm{VT}_{1}\right)$ and regulatory parameters (kinetics) at muscular level. 


\section{Heart rate-mean values and regulatory aspects}

In comparison with the pre and post-tests, mean HR values during the constant phases of the WR protocol during the mission phase were lower. However, HR kinetics at MD-8 were slower compared with MD22, MD42 and $\mathrm{MD}+4$. Hence, changes in the absolute HR values during the WR step and the speed of adjustment of HR (kinetics) in response to increased metabolic demands are not similar. Regarding the altered regulatory behavior of HR, a training effect of the exercise countermeasure can be concluded, which is not evident in the mean values of HR. Therefore, two aspects should be considered: (1) a training effect via the exercise countermeasure and (2) autonomic system changes caused by reduced external stimuli. Regarding the effect of the exercise countermeasure (1), the accelerated HR kinetics starting at MD22 are in accordance with the increased WR at VT1 after the end of the mission (MD+4). For comparison, after bed rest, HR kinetics were significantly slowed as a result of deconditioning (Koschate et al. 2018) and were correlated with changes in $\mathrm{V}_{2 \text { peak }}$ in astronauts before and after their mission to the International Space Station (Hoffmann et al. 2016). After 12 weeks of endurance exercise training, HR kinetics were accelerated in a group of male type 2 diabetes patients (Koschate et al. 2016a). Hence, HR kinetics seem to be a sensitive parameter to observe changes in cardiovascular regulation in response to exercise training stimuli. These training effects were not visible in the mean values of HR during the exercise test throughout the HERA missions. Considering the potential changes in the autonomic nervous system due to the confinement phase (2), either a higher parasympathetic system activity during the mission or an increased sympathetic system activity before and after the mission (Coote 2010) should be considered, which might (although not statistically significant between MD42 and MD+4) also slightly dampen the potential training effect, which was observed for HR kinetics at MD42. During Russian confinement experiments with durations of 120 and $520 \mathrm{~d}$ (Vigo et al. 2012, 2013), a greater HRV and reduced cortical activation levels were reported (Weber et al. 2020; Jacubowski et al. 2015). Greater HRV was suggested to be influenced by boredom, artificial light or greater parasympathetic system activity during isolation (Vigo et al. 2012, 2013). These circumstances might have altered the exercise HR during the HERA C4 missions as well.

In experiments, applying acute and chronic (partial) sleep deprivation, reduced HR variability, higher HR, increases in sympathovagal activity and decreases in vagal activity in the resting condition and/or during sleep were described (Tobaldini et al. 2017a, b; Dettoni et al. 2012; Glos et al. 2014; Fogt et al. 2011; Liu et al. 2015). Dettoni et al. (2012) found increased sympathetic activity in the resting supine position after only five nights of partial sleep restriction, which is comparable to the HERA campaign (5 h). Using linear mixed-effects models, significant linear relationships were found between fatigue level and HRV (Fogt et al. 2011). This is in contrast to the observed cardiovascular data during exercise in this experiment. The findings of the present experiment, supported by the data of Vigo et al. (2012, 2013) suggest, that the effect of confinement and reduced external stimuli in combination with the applied exercise countermeasure during the simulated mission to an asteroid might have had a greater effect on the cardiovascular system, than partial sleep deprivation. Therefore, partial sleep deprivation, at least when combined with the applied exercise countermeasure does not yield negative effects regarding the regulation of the cardiovascular system at moderate WR intensities. In line with this, no correlations were found between baseline $\dot{\mathrm{VO}}_{2 \text { peak }}, \dot{\mathrm{VO}}_{2 \text { musc }}$ or pre to post changes in these parameters with HR kinetics.

\section{Limitations}

No further parameters to explain the lower HRs during the mission compared with the pre and post mission values were assessed. Therefore, it remains unclear whether the sympathetic nervous system activity was increased before or decreased during the mission and vice versa, whether the parasympathetic system activity was higher during or lower before and after the mission. The effect of confinement on cardiorespiratory fitness, cannot be evaluated separately from the effect of sleep restrictions, since no test during the mission was performed after recovery sleep.

Two participants had to terminate the test early, because they exceeded threshold values for HR or blood pressure, which were set by the NASA medical board. Hence, for future confinement experiments, a further reduction of the applied WR intensities should be considered.

\section{Conclusions}

The kinetics of HR were significantly faster during and at the end of the simulated space mission including sleep deprivation, compared to the values before the mission. Therefore, HR kinetics seem to indicate changes in cardiovascular regulation very sensitively. Absolute values of HR during the exercise test followed a different timeline, with higher values before and after the mission. Changes in $\mathrm{VO}_{2 \text { musc }}$ kinetics and $\mathrm{V}_{2 \text { peak }}$ were not significant, but less fit individuals seemed to benefit more from the applied exercise training countermeasure, than crew members with a high baseline fitness level. Hence, for future missions, the exercise countermeasure protocol should be intensified for people with a higher fitness status. The applied exercise test seems promising for the evaluation of the cardiovascular and respiratory 
system during circumstances where medical support is not consistently available, or in patients where maximal exercise intensities are not suitable, since it requires only moderate WR intensities and only a short amount of time. Especially, HR kinetics should be investigated in more detail. The kinetics of HR are simple to assess during exercise and provide more sophisticated information about the status of the cardiovascular system, than absolute values of HR alone.

Acknowledgements This research was supported by a research fund of the German Federal Ministry for Economic Affairs and Energy/ German Aerospace Center (DLR e.V.; FKZ: 50WB1626). HERA Campaign 4 was organized by NASA and conducted at Lyndon B. Johnson Space Center in Houston, Texas, USA. We thank all crew members for participation, the NASA HERA team for the organization of the study and coordination of the experiments, and their support with the transport and onsite handling of the equipment and all documents. We would also like to thank Lutz Thieschäfer, Janosch Wacker and Zeynep Acar for their support with the preparation of the experiment and data processing.

Author's contributions JK, UH and UD designed the work, JK acquired the data, JK, UH and UD analysed and discussed the results. JK drafted the manuscript, UH and UD critically revised and approved the final version of the manuscript. All authors agree to be accountable for all aspects of the work in ensuring that questions related to the accuracy or integrity of any part of the work are appropriately investigated and resolved. All persons designated as authors qualify for authorship, and all those who qualify for authorship are listed.

Funding Open Access funding enabled and organized by Projekt DEAL. This research was supported by a research fund of the German Federal Ministry for Economic Affairs and Energy/German Aerospace Center (DLR e.V.; FKZ: 50WB1626).

Data availability Data available upon request due to privacy/ethical restrictions.

\section{Declarations}

Conflict of interest The authors declare, that they have no conflict of interest to report.

Ethical approval Ethical approval was obtained from the Institutional Review Board at the NASA Johnson Space Center (Protocol number: Pro2320) as well as the Ethical Committee of the German Sport University Cologne (Protocol number: 074/2016) in accordance with the Declaration of Helsinki (including its amendments until 2013).

Consent to participate Written informed consent was available from all participants ahead of the experiments.

Open Access This article is licensed under a Creative Commons Attribution 4.0 International License, which permits use, sharing, adaptation, distribution and reproduction in any medium or format, as long as you give appropriate credit to the original author(s) and the source, provide a link to the Creative Commons licence, and indicate if changes were made. The images or other third party material in this article are included in the article's Creative Commons licence, unless indicated otherwise in a credit line to the material. If material is not included in the article's Creative Commons licence and your intended use is not permitted by statutory regulation or exceeds the permitted use, you will need to obtain permission directly from the copyright holder. To view a copy of this licence, visit http://creativecommons.org/licenses/by/4.0/.

\section{References}

Barger LK, Flynn-Evans EE, Kubey A, Walsh L, Ronda JM, Wang W, Wright KP, Czeisler CA (2014) Prevalence of sleep deficiency and use of hypnotic drugs in astronauts before, during, and after spaceflight: an observational study. Lancet Neurol 13(9):904-912. https://doi.org/10.1016/S1474-4422(14)70122-X

Beaver WL, Wasserman K, Whipp BJ (1986) A new method for detecting anaerobic threshold by gas exchange. J Appl Physiol 60(6):2020-2027

Benjamini Y, Hochberg Y (1995) Controlling the false discovery rate: a practical and powerful approach to multiple testing. J Royal Stat Soc Series B (methodological) 57(2):289-300

Bennett FM, Reischl P, Grodins FS, Yamashiro SM, Fordyce WE (1981) Dynamics of ventilatory responses to exercise in humans. J Appl Physiol Respirat Environ Exercise Physiol 51(1):194-203

Bennett BL, Schlichting CL, Bondi KR (1985) Cardiorespiratory fitness and cognitive performance before and after confinement in a nuclear submarine. Aviat Space Environ Med 56(11):1085-1091

Buchheit M, Simon C, Charloux A, Doutreleau S, Piquard F, Brandenberger G (2005) Heart rate variability and intensity of habitual physical activity in middle-aged persons. Med Sci Sports Exerc 37(9):1530-1534. https://doi.org/10.1249/01.mss.0000177556. 05081.77

Coote JH (2010) Recovery of heart rate following intense dynamic exercise. Exp Physiol 95(3):431-440. https://doi.org/10.1113/ expphysiol.2009.047548

De La Torre GG, van Baarsen B, Ferlazzo F, Kanas N, Weiss K, Schneider S, Whiteley I (2012) Future perspectives on space psychology: recommendations on psychosocial and neurobehavioural aspects of human spaceflight. Acta Astronaut 81(2):587-599. https://doi.org/10.1016/j.actaastro.2012.08.013

Dettoni JL, Consolim-Colombo FM, Drager LF, Rubira MC, de Souza SBPC, Irigoyen MC, Mostarda C, Borile S, Krieger EM, Moreno H, Lorenzi-Filho G (2012) Cardiovascular effects of partial sleep deprivation in healthy volunteers. J Appl Physiol 113(2):232-236. https://doi.org/10.1152/japplphysiol.01604.2011

Drescher U, Koschate J, Hoffmann U (2015) Oxygen uptake and heart rate kinetics during dynamic upper and lower body exercise: an investigation by time-series analysis. Eur J Appl Physiol 115(8):1665-1672. https://doi.org/10.1007/s00421-015-3146-4

Fogt DL, Cooke WH, Kalns JE, Michael DJ (2011) Linear mixedeffects modeling of the relationship between heart rate variability and fatigue arising from sleep deprivation. Aviat Space Environ Med 82(12):1104-1109

Fomina EV, Lysova NY, Kukoba TB, Grishin AP, Kornienko MB (2017) One-year mission on ISS is a step towards interplanetary missions. Aerosp Med Human Perform 88(12):1094-1099. https:// doi.org/10.3357/AMHP.4847.2017

Glos M, Fietze I, Blau A, Baumann G, Penzel T (2014) Cardiac autonomic modulation and sleepiness: physiological consequences of sleep deprivation due to $40 \mathrm{~h}$ of prolonged wakefulness. Physiol Behav 125:45-53. https://doi.org/10.1016/j.physbeh.2013.11.011

Grassi B (2000) Skeletal muscle V O2 on-kinetics: set by O2 delivery or by $\mathrm{O} 2$ utilization? New insights into an old issue. Med Sci Sports Exerc 32(1):105-116 
Grassi B (2006) Oxygen uptake kinetics: why are they so slow? And what do they tell us? J Physiol Pharmacol 57:53-65

Hawkins MN, Raven PB, Snell PG, Stray-Gundersen J, Levine BD (2007) Maximal oxygen uptake as a parametric measure of cardiorespiratory capacity. Med Sci Sports Exerc 39(1):103-107. https://doi.org/10.1249/01.mss.0000241641.75101.64

Hoffmann U, Drescher U, Benson AP, Rossiter HB, Essfeld D (2013) Skeletal muscle $\mathrm{VO}_{2}$ kinetics from cardio-pulmonary measurements: assessing distortions through $\mathrm{O}(2)$ transport by means of stochastic work-rate signals and circulatory modelling. Eur J Appl Physiol 113(7):1745-1754. https://doi.org/10.1007/ s00421-013-2598-7

Hoffmann U, Moore AD, Koschate J, Drescher U (2016) $\mathrm{VO}_{2}$ and HR kinetics before and after International Space Station missions. Eur J Appl Physiol 116(3):503-511. https://doi.org/10.1007/ s00421-015-3298-2

Hughson RL, Tschakovsky ME, Houston ME (2001) Regulation of oxygen consumption at the onset of exercise. Exerc Sport Sci Rev 29(3): 1

Jacubowski A, Abeln V, Vogt T, Yi B, Choukèr A, Fomina E, Strüder HK, Schneider S (2015) The impact of long-term confinement and exercise on central and peripheral stress markers. Physiol Behav 152(Pt A):106-111. https://doi.org/10.1016/j.physbeh.2015.09. 017

Jouanin J-C, Dussault C, Pérès M, Satabin P, Piérard C, Guézennec CY (2004) Analysis of heart rate variability after a ranger training course. Mil Med 169(8):583-587. https://doi.org/10.7205/ milmed.169.8.583

Katzel LI, Sorkin JD, Fleg JL (2001) A comparison of longitudinal changes in aerobic fitness in older endurance Athletes and sedentary men. J Am Geratr Soc 49:1657-1664

Koschate J, Drescher U, Thieschäfer L, Heine O, Baum K, Hoffmann U (2016b) Cardiorespiratory kinetics determined by pseudorandom binary sequences - comparisons between walking and cycling. Int J Sports Med 37(14):1110-1116. https://doi.org/10. 1055/s-0042-114702

Koschate J, Drescher U, Brinkmann C, Baum K, Schiffer T, Latsch J, Brixius K, Hoffmann U (2016a) Faster heart rate and muscular oxygen uptake kinetics in type 2 diabetes patients following endurance training. Appl Physiol Nutr Metab 41(11):1146-1154. https://doi.org/10.1139/apnm-2016-0001

Koschate J, Thieschäfer L, Drescher U, Hoffmann U (2018) Impact of 60 days of $6^{\circ}$ head down tilt bed rest on muscular oxygen uptake and heart rate kinetics: efficacy of a reactive sledge jump countermeasure. Eur J Appl Physiol 118(9):1885-1901. https://doi.org/ 10.1007/s00421-018-3915-y

Liu Q, Zhou R, Liu L, Zhao X (2015) Effects of 72 hours total sleep deprivation on male astronauts' executive functions and emotion. Compr Psychiatry 61:28-35. https://doi.org/10.1016/j.comppsych. 2015.05.015

Ludwig M, Hoffmann K, Endler S, Asteroth A, Wiemeyer J (2018) Measurement, prediction, and control of individual heart rate responses to exercise-basics and options for wearable devices. Front Physiol 9:778. https://doi.org/10.3389/fphys.2018.00778

Mairesse O, MacDonald-Nethercott E, Neu D, Tellez HF, Dessy E, Neyt X, Meeusen R, Pattyn N (2019) Preparing for Mars: human sleep and performance during a 13 month stay in Antarctica. Sleep 42(1). https://doi.org/10.1093/sleep/zsy206

Moore AD, Downs ME, Lee SMC, Feiveson AH, Knudsen P, PloutzSnyder L (2014) Peak exercise oxygen uptake during and following long-duration spaceflight. J Appl Physiol 117(3):231238. https://doi.org/10.1152/japplphysiol.01251.2013

Moraes MM, Mendes TT, Martins YAT, Espinosa CN, Maluf CB, Soares DD, Wanner SP, Arantes RME (2018) The changes in maximal oxygen uptake (VO2MAX) induced by physical exertion during an Antarctic expedition depend on the initial VO2MAX of the individuals: a case study of the Brazilian expedition. Int J Circumpolar Health 77(1):1521244. https://doi.org/10.1080/ 22423982.2018.1521244

Myers J, Forman DE, Balady GJ, Franklin BA, Nelson-Worel J, Martin B-J, Herbert WG, Guazzi M, Arena R (2014) Supervision of exercise testing by nonphysicians: a scientific statement from the American Heart Association. Circulation 130(12):1014-1027. https://doi.org/10.1161/CIR.0000000000000101

Norsk P (2020) Adaptation of the cardiovascular system to weightlessness: surprises, paradoxes and implications for deep space missions. Acta Physiol (oxford, England) 228(3):e13434. https:// doi.org/10.1111/apha.13434

Poole DC, Jones AM (2017) Measurement of the maximum oxygen uptake Vo2max: Vo2peak is no longer acceptable. J Appl Physiol 122(4):997-1002. https://doi.org/10.1152/japplphysiol.01063. 2016

Rossiter HB (2011) Exercise: kinetic considerations for gas exchange. Compr Physiol 1(1):203-244. https://doi.org/10.1002/cphy.c0900 10

Strangman GE, Sipes W, Beven G (2014) Human cognitive performance in spaceflight and analogue environments. Aviat Space Environ Med 85(10):1033-1048. https://doi.org/10.3357/ASEM. 3961.2014

Tobaldini E, Costantino G, Solbiati M, Cogliati C, Kara T, Nobili L, Montano N (2017a) Sleep, sleep deprivation, autonomic nervous system and cardiovascular diseases. Neurosci Biobehav Rev 74(Pt B):321-329. https://doi.org/10.1016/j.neubiorev.2016.07.004

Tobaldini E, Covassin N, Calvin A, Singh P, Bukartyk J, Wang S, Montano N, Somers VK (2017b) Cardiac autonomic control and complexity during sleep are preserved after chronic sleep restriction in healthy subjects. Physiol Rep 5(7):e13197. https://doi.org/ 10.14814/phy2.13197

Vigo DE, Ogrinz B, Wan L, Bersenev E, Tuerlinckx F, van der Bergh O, Aubert AE (2012) Sleep-wake differences in heart rate variability during a 105-day simulated mission to Mars. Aviat Space Environ Med 83(2): 125-130

Vigo DE, Tuerlinckx F, Ogrinz B, Wan L, Simonelli G, Bersenev E, van den Bergh O, Aubert AE (2013) Circadian rhythm of autonomic cardiovascular control during Mars500 simulated mission to Mars. Aviat Space Environ Med 84(10):1023-1028. https://doi. org/10.3357/asem.3612.2013

Weber J, Klein T, Abeln V (2020) Shifts in broadband power and alpha peak frequency observed during long-term isolation. Sci Rep 10(1):17987. https://doi.org/10.1038/s41598-020-75127-0

Yi B, Matzel S, Feuerecker M, Hörl M, Ladinig C, Abeln V, Choukèr A, Schneider S (2015) The impact of chronic stress burden of 520-d isolation and confinement on the physiological response to subsequent acute stress challenge. Behav Brain Res 281:111-115. https://doi.org/10.1016/j.bbr.2014.12.011

Publisher's Note Springer Nature remains neutral with regard to jurisdictional claims in published maps and institutional affiliations. 